\title{
Aplicação de metodologia para o desenvolvimento do sistema computacional MESO a luz da usabilidade
}

\author{
Fábio Josende $\mathrm{Paz}^{1}$ \\ Liane Mahlmann Kipper ${ }^{2}$ \\ Rejane Frozza ${ }^{2}$ \\ William Joseph Giraldo Orozco ${ }^{3}$
}

\begin{abstract}
Resumo: Modelos de avaliação são utilizados em diversas áreas empresariais, porém muitos caem em desuso, ou são substituídos pela sua complexidade ou pela dificuldade de utilização. O sistema computacional Maturidade e Educação para a Sustentabilidade Organizacional - MESO tem a intenção de ser simples e de fácil utilização, além de ser on-line. Neste contexto, observa-se a necessidade de buscar uma metodologia que contenha princípios da usabilidade para a criação de um sistema computacional. O objetivo é identificar se as técnicas de usabilidade podem auxiliar no desenvolvimento do sistema computacional MESO tornando-o mais utilizável aos usuários. Para isto, foram realizadas uma pesquisa bibliográfica e a aplicação da metodologia TD-MBUID que contempla técnicas de usabilidade para a modelagem e testes em sistemas. Os resultados encontrados evidenciam a importância de aliar técnicas de usabilidade no desenvolvimento de softwares e que os testes realizados com o aplicativo Tobii Studio comprovaram que a utilização dos modelos mentais dos usuários para a elaboração de interfaces melhora a usabilidade e a interação dos usuários com as mesmas.
\end{abstract}

Palavras-chave: Ferramentas computacionais, TD-MBUID, Usabilidade.

\begin{abstract}
Evaluation models are used in various business areas, but many fall into disuse, or are replaced by their complexity or the difficulty of use. The computational of Maturity and Education for Sustainability -MESO is intended to be simple and easy to use, in addition to being online. In this context, the need to find a methodology that contains principles of usability to the creation of a computer system. The aim is to identify if the usability techniques can assist in the development of computational MESO system making it more usable to users. For this, a bibliographical research and application of TD-MBUID methodology which includes usability techniques for modeling and systems testing. The results demonstrate the importance of combining usability techniques in software development and that the tests conducted with the Tobii Studio application proved that the use of the users' mental models for the development of interfaces and usability improves the interaction of users with the same.
\end{abstract}

Key words: Computational tools, TD-MBUID, Usability.

\footnotetext{
${ }^{1}$ Universidade da Região da Campanha, Departamento Ciência da Computação, Campus central, Av. Tupy Silveira, 2099, Cep 96400-110. Bagé - RS / Brasil

fabiojpaz@gmail.com

${ }^{2}$ Universidade de Santa Cruz do Sul, Programa de Pós-Graduação em Sistemas e Processos Industriais. Av. Independência, 2293. Cep 96815-900. Santa Cruz do Sul - RS / Brasil.

\{liane@unisc.br, frozza@unisc.br\}

${ }^{3}$ Universidade del Quindio, Grupo de Inverstigação de Sistemas de Informação e Controle Industriais, Carrera 15 Calle 12 Norte, Armênia - Colômbia.

wjgiraldo@uniquindio.edu.co
}

http://dx.doi.org/10.5335/rbca.v9i3.6171 


\section{Introdução}

Criar softwares ou sites atraentes aos usuários é uma missão complicada, principalmente se não forem utilizadas técnicas que permitam avaliar ou medir a satisfação dos usuários na sua utilização. Carneiro e Ishitani [1] comentam que em um dos seus estudos observou-se que se faz também necessário explorar a interação entre usuários de um ambiente voltado para a educação. O estudo da usabilidade surgiu com esta intenção, criando regras que procurem orientar os processos de desenvolvimento de sistemas ou sites para que estes se tornem eficientes em seus propósitos, eficazes do ponto de vista dos clientes ou usuários, seguros e principalmente confortáveis em sua utilização. Nielsen [2] afirma que o maior objetivo de todos esses princípios é facilitar a interação do usuário com o produto. Bevan [3] e Lima et al. [4] consideram a usabilidade como a habilidade do usuário em realizar uma determinada tarefa com sucesso. Porém Bevan [3]; Ferreira e Reis [5] e Park et al., [6] afirmam também que existem muitas organizações que não utilizam avaliações de usabilidade durante o ciclo de desenvolvimento de seus produtos, principalmente nas fases iniciais do seu desenvolvimento.

Na criação do sistema computacional para avaliar a Maturidade e Educação para a Sustentabilidade Organizacional - MESO desejou-se utilizar estes princípios, no entanto, neste sistema o foco da usabilidade está na construção do questionário e em como gerar intenção no usuário para respondê-lo, ou seja, em utilizar o sistema. O foco está na simplicidade e facilidade da sua interface, gerando intenção de uso, sem a necessidade de profissionais treinados para sua aplicação, facilitando seu uso por empresas de todos os portes, mas principalmente em relação às micros e pequenas empresas.

Neste contexto, este artigo tem como objetivo identificar se técnicas de usabilidade podem auxiliar no desenvolvimento do sistema computacional MESO. Para isto foram realizados estudos teóricos e a aplicação de uma metodologia que aponta para importância de aplicar estas técnicas de usabilidade para que o sistema seja bem elaborado, tanto em sua criação conceitual como no seu design. A seguir, na seção 2, apresenta-se um breve referencial teórico, resultante da pesquisa bibliográfica realizada, sobre os temas usabilidade e a descrição da metodologia TD-MBUID, proposta por Orozco [7]. Na seção 3 está descrita uma síntese da metodologia utilizada, seguida pelos principais resultados, considerações finais e referências.

\section{Estudos teóricos e metodológicos}

Esta seção apresenta os principais conceitos referentes aos temas usabilidade, técnicas utilizadas para desenvolvimento de interfaces e a metodologia TD-MBUID.

\subsection{Usabilidade}

Usabilidade é um atributo de qualidade relacionado à facilidade em usar algo, ou seja, a rapidez com que os usuários podem aprender a usar alguma coisa (Norman [8]; Nielsen e Loranger [9]; Silvia [10]; Lima et al. [4] e Nielsen [2]). Na Web, a usabilidade é uma condição para a sobrevivência do site, ou seja, se for difícil de usar o site o usuário simplesmente o abandona. Já em uma página de intranet a usabilidade é uma questão de produtividade dos colaboradores, pois tempo perdido para ler instruções ou encontrar algo significa dinheiro perdido [2].

Assim, Preece et al. [11]; Nielsen e Loranger [9] e Ferreira e Reis [5], reforçam que é necessário projetar para o usuário, ou seja, escutar os usuários, tornando-os centro de seu projeto. Projetar interfaces elaboradas e não convencionais pode gerar resultados esteticamente bonitos, porém a chave para um bom sistema é a simplicidade aliada a um design. Preece et al. [11] e Bevan et al. [12] afirmam a importância em testar o sistema com usuários reais, dando-lhes tarefas e observá-los, esse é o teste mais eficiente, fácil e barato de fazer. Nielsen [2] afirma que testar com cinco usuários já é o suficiente e ainda complementa que ouvi-los é enganador, o importante é observá-los na realização das tarefas.

Bevan [13] e Martins et al., [14] vão além e afirmam que a usabilidade permite contemplar aspectos como diversão, eficácia, estética, bem-estar e suporte para o desenvolvimento humano entre outros. Já Quaresma e Moraes [15] em seus estudos demonstram a importância da usabilidade para medir a satisfação de uso de interfaces. Portanto, percebe-se que a melhoria da usabilidade apresenta muitos benefícios os quais Bevan et. al. [12] e Park et al., [16] em seus estudos citam: 
- Aumento da eficácia e da eficiência: Um sistema adaptado ao modo como o utilizador age permite uma interação mais eficaz e eficiente.

- Maior produtividade: Permite que o utilizador se concentre na tarefa e não na ferramenta, aumentando o seu desempenho em consequência da qualidade da interação.

- Menor necessidade de formação: Um sistema com um bom nível de usabilidade, projetado com base no utilizador final facilita a aprendizagem.

- Melhoria da aceitação: Gera maior confiança sistemas bem projetados com funcionalidades que facilitem a informação para o usuário.

- Apoio a utilizadores com menos competências tecnológicas.

- Redução de erros.

Resumindo, usabilidade é percebida quando se analisa como o usuário utiliza um determinado sistema e como estão organizadas as tarefas que ele realiza, ou seja, conhecer como as outras tarefas afetam o trabalho do usuário e entender a melhor maneira de apresentar o ambiente para o mesmo. É interessante que o ambiente apresente alguns atrativos e benefícios que façam o usuário utilizar o sistema. Portanto, a utilização de técnicas de usabilidade no sistema proposto é para melhor desenhar o ambiente composto principalmente pela análise do formulário de pesquisa junto ao usuário como também na busca de uma melhor forma de apresentação (design) aos seus usuários.

Para avaliar a usabilidade Nielsen e Mack [17] e Nielsen e Loranger [9] dividem em quatro categorias ou formas de avaliação, conforme se apresenta a seguir:

a) Automática: As especificações listadas para a interface analisada e o resultado final sofrem um processo comparativo por meio de softwares projetados com esse fim.

b) Empiríca: Funciona por meio de observação. A usabilidade da interface é posta à prova por meio de testes com os usuários reais. É a forma de avaliação mais utilizada, mas ainda apresenta um custo elevado.

c) Formal: A usabilidade é medida a partir de modelos e fórmulas. Apresenta dificuldades de aplicação, principalmente com interfaces altamente interativas e complexas.

d) Informal: Baseadas em regras heurísticas e de experiências, conhecimentos ou habilidades pessoais do avaliador ou de grupo interdisciplinares.

Segundo Nielsen [2], o ideal é possuir um laboratório para testes, porém uma sala ou escritório que não possuam distrações funciona perfeitamente, mas o que realmente importa é que o usuário fique à disposição para ser observado enquanto interage com o design e o sistema.

\subsection{Técnicas e metodologias utilizadas para desenvolvimento de interfaces}

Existem diversas metodologias de desenvolvimento de softwares, porém as mais utilizadas, segundo Pressman [18] e Sommerville [19], são as metodologias ágeis como Scrum e XP. Nestas o foco deixa de ser a documentação e definição completa do sistema e passa a ser a colaboração do cliente e a interação, gerando softwares executáveis de uma forma mais rápida, porém nenhuma destas metodologias tem como prioridade a usabilidade do sistema a ser construído, levando em consideração seus usuários.

Corroborando, Nascimento et al. [20] realizaram uma avaliação da usabilidade e UX (User Experience) em um aplicativo educacional desenvolvido com metodologia ágil. Os resultados encontrados revelam que muitos problemas foram detectados e melhorias foram sugeridas, permitindo assim uma nova versão do aplicativo.

Barbora et al. [21] realizou um estudo sobre a XPLUS que é uma metodologia centrada no usuário a qual se propõe a inserir técnicas de design e usabilidade nas principais fases do ciclo iterativo da metodologia XP (eXtreme Programming), ainda que os autores acreditem que a XPLUS represente um avanço em relação a outras metodologias ágeis ao reconhecer a importância do design e da usabilidade no desenvolvimento de software, ela apenas sugere a aplicação de técnicas de maneira muito sucinta e simples.

Nesse sentido, uma metodologia que se preocupe com inspeções de usabilidade e UX como medida preventiva no desenvolvimento de software podem trazer bons resultados a todo o processo. No período de estudos na Universidad del Quindío na Colômbia foi possível conhecer várias técnicas que o Grupo de Investigação de Sistemas de Informação e Controle Industriais - SINFOCI utiliza para o desenvolvimento de 
seus projetos e a metodologia escolhida foi a Task \& Data - Model Based User Interface Development (TDMBUID) encontrada na tese de doutorado "Marco de Desarrollo de Sistemas Groupware Interactivos Basado en la Integración de Procesos y Notaciones" de Orozco [7]. Esta escolha justifica-se pelo fato da metodologia considerar a modelagem a partir dos formulários em papel, utiliza as técnicas de usabilidade descritas por Nielsen [17] de maneira preventiva, além de ser centrada nos modelos mentais do usuário o que a diferencia de metodologias tradicionais e ágeis.

\section{Descrição da metodologia Task \& Data - Model Based User Interface Development (TD-MBUID) - Orozco (2010) [7]}

O desenvolvimento da interface de usuário pode ser visto na figura 1, dividido em dois níveis de modelagem. Inicialmente, durante a modelagem do sistema de negócio, são analisadas as tarefas interação (entre pessoas) e objetos de domínio (entidades de negócio), que ficam independentes da tecnologia e nos permite compreender os modelos mentais dos usuários para desenvolver a interface do usuário do negócio a partir de dados de negócios. A modelagem da interface do usuário do negócio é feita inicialmente através de formulários em papel, que suporta a realidade e independe de tecnologia (sem botões). Após, durante o desenvolvimento do sistema interatico, são analisadas as tarefas interativas (entre o usuário e o sistema) e objetos (classes de design) para identificar os diálogos entre o usuário e o computador. Este diálogo acontece através de um conjunto de interfaces que são geradas a partir de uma especificação abstrata para, por fim, obter um resultado.

Ao contrário da maioria das abordagens de desenho, este método propõe especificar a interação com o sistema depois de desenhadas as interfaces de negócio do usuário. Esta proposta de desenvolvimento de interface de usuário dá liberdade ao desenvolvedor de continuar o desenho a partir dos dados iniciais e das tarefas interativas, porém esse processo não é completamente automático e requer a intervenção constante do usuário, criando um forte vínculo de participação do usuário no processo de desenvolvimento da interface.

Figura 1: Fluxo de desenvolvimento de interface de usuário na metodologia TD-MBUID

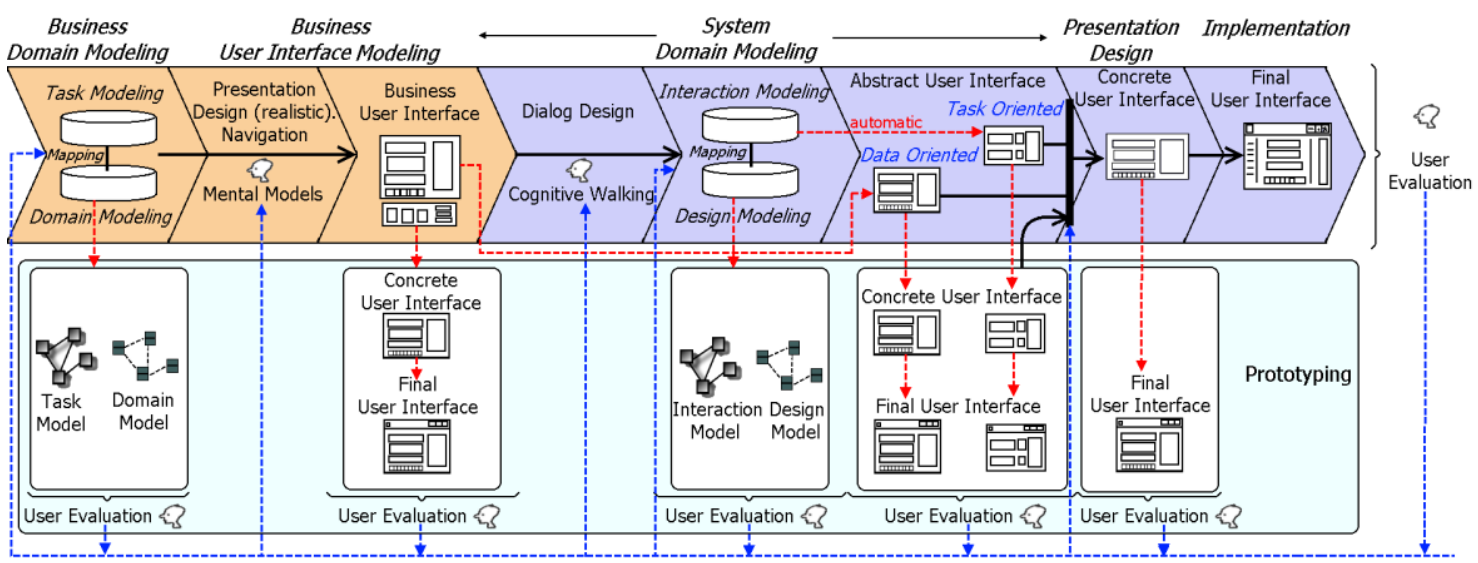

Fonte: Adaptado de Orozco [7].

A figura 1 demonstra o fluxo do desenvolvimento descrito na direção do eixo X, por outro lado percebese outra dimensão, no eixo y. Isso define diferentes fluxos de desenvolvimento de interface de usuário o que possibilita avaliar o desenho na medida em que evolui o ciclo de vida de desenvolvimento do processo.

TD-MBUID é uma metodologia de desenho centrada no usuário (DCU). Uma metodologia DCU é aquela em o usuário se envolve em todas as fases do desenvolvimento, na figura 1 se observa como o usuário tem uma grande relevância na captura da informação mais importante que está associada ao fator humano. TD-MBUID propõe um método sistemático no qual, a subjetividade do desenvolvedor e a subjetividade do usuário encontram-se representadas em todos os artefatos do desenho. Utilizando TD-MBUID no desenvolvimento de produtos, ambos, o desenvolvedor e o usuário conseguem produzir sistemas que tenham as características de ambos, "pode-se dizer que um produto feito o TD-MBUID é como um filho que se aparece tanto com sua mãe (desenvolvedor: dá ou fornece informações e cria), quanto seu pai (usuário: da ou fornece informações)." Neste sentido, o produto não pode ser negado nem por um, nem por outro [22]. Na fase de modelagem abstrata da interface o desenvolvedor pode gerar protótipos de interface seguindo dois tipos de rotas: a rota baseada em 
dados (a partir da interface de negócio do usuário) ou a rota baseada em tarefas (a partir do modelo de interação), conforme figura 2.

Figura 2: Processo de modelagem TD-MBUID

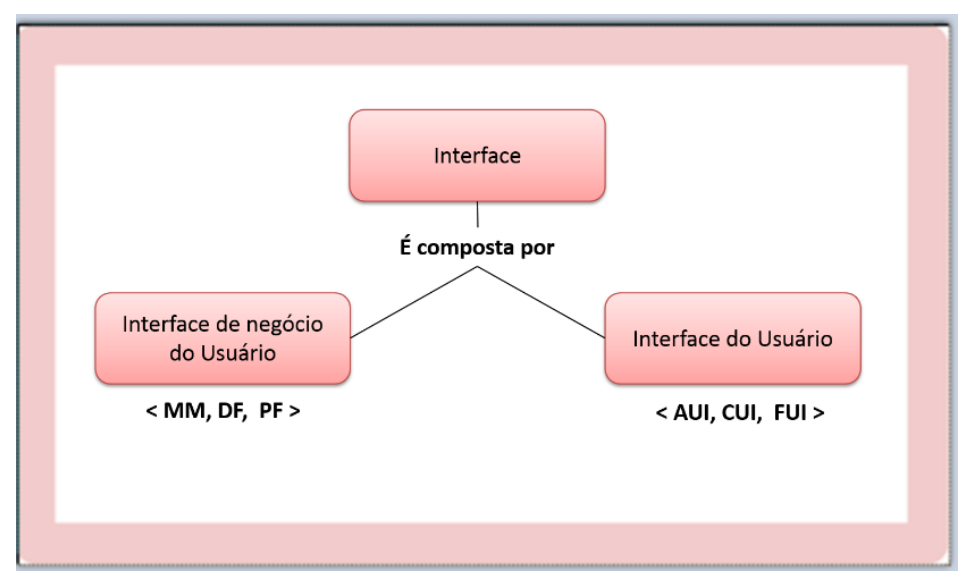

Fonte: Adaptado de Orozco [7].

Na figura 2, observa-se a interface do usuário do negócio (Business User Interface) que é a interface em papel que representa a captura da informação dos processos de negócio, ou seja, a informação que é compartilhada entre usuários e trabalhadores em cada um de seus atos comunicativos. Esta interface do usuário do negócio é independente da tecnologia e neste nível se encontra os Modelos Mentais (MM - Mental Models), Formulário de dados (DF - Data Form) e formulário de papel (PF - Paper Form). Também a Interface de Usuário (User Interface) onde se encontra a interface abstrata de usuário (AUI - Abstract User Interface), a interface concreta de usuário (CUI - Concret User Interface) e a interface final do usuário (FUI - Final User Interface).

Para o desenvolvimento de qualquer sistema utilizando a metodologia proposta, é necessário um plano de usabilidade que, segundo Orozco [7] precisa verificar qual ou quais os ambientes (locais de uso) em que o sistema será utilizado, as plataformas computacionais que serão usadas, por exemplo, intranet da organização, internet, entre outros, quais os tipos de usuários e as tarefas que poderão ser realizadas. A figura 3 apresenta a ideia descrita por Orozco [7]. Segundo Nielsen e Loranger [9] e Orozco [7], a usabilidade está intrínseca quando se analisa as tarefas e como está composto o trabalho, por isso existe a necessidade de se identificar e compreender as tarefas que usuário irá fazer, ou seja, analisar a semântica do trabalho (como se chamam as tarefas e a sua influência no formulário, quem é o autor principal, como o trabalho é finalizado, como gerar intenção no usuário, entre outras).

Figura 3: Plano inicial de usabilidade

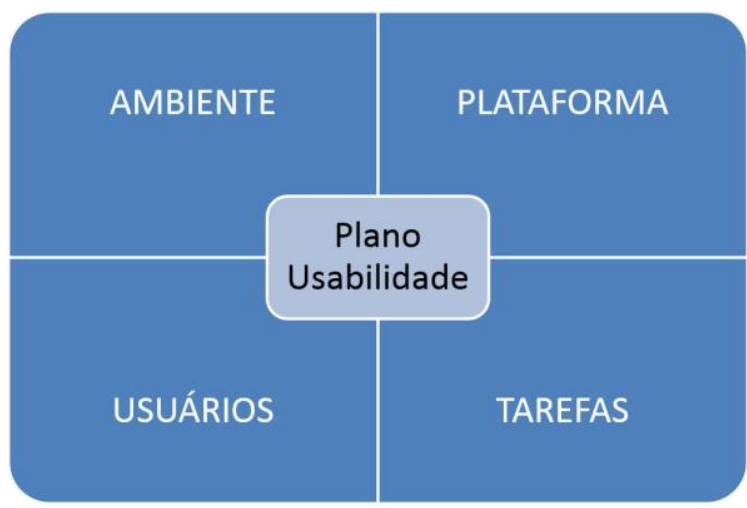

Fonte: Adaptado de Orozco [7].

Para a aplicação da metodologia é necessário conhecer alguns conceitos da TD-MBUID para melhor entendimento da figura 4. São oito os conceitos e os mesmos estão descritos a seguir. 
- Domínio do problema de negócio: É a situação problema modelada em torno do trabalho do usuário.

- Prototipagem: É o processo de criação de um esboço da interface do usuário, com a finalidade de explorar e validar o desenho antes de iniciar o desenvolvimento real.

- Dados do domínio de negócio: Este componente ilustra a estrutura dos dados do domínio do negócio, representados por entidades de negócio.

- Usuários chaves: São as pessoas que possuem o conhecimento sobre os dados do negócio.

- Desenvolvedores: São as pessoas encarregadas de desenvolver a interface do usuário.

- Modelos mentais: Este componente representa o desenho da interface de negócio do usuário, criada em papel e que representa o entendimento do usuário e as peças chaves dos dados do modelo de domínio.

- Formulário de dados: Este componente representa o desenho da estrutura dos dados de acordo como a informação é exibida na interface.

- Formulário em papel: Este componente representa o desenho dos formulários de maneira realista.

Figura 4: Imagem descritiva da aplicação da metodologia TD-MBUID.

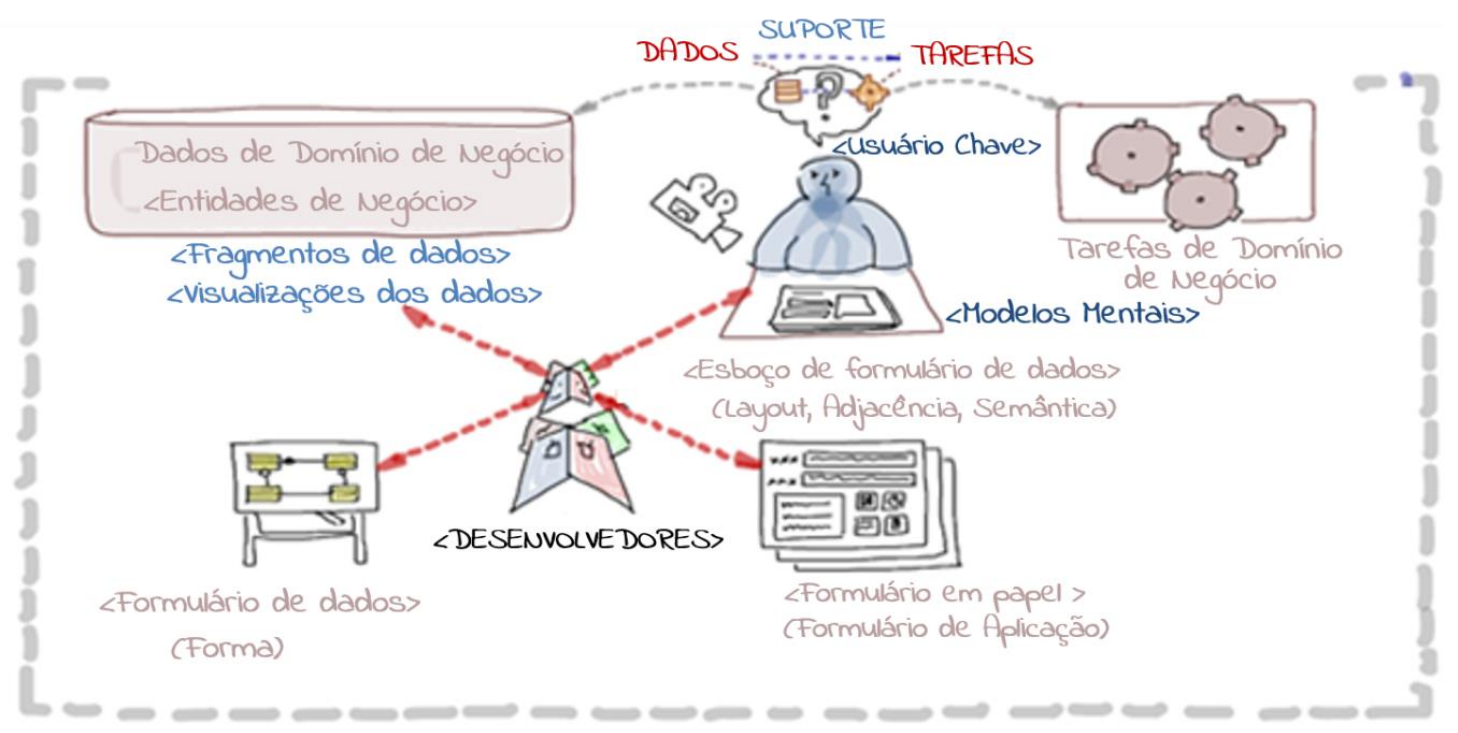

Fonte: Adaptado de Orozco [7].

A Figura 4 apresenta o usuário chave, o qual representa um projeto inicial da interface do usuário, que pode conter o modelo de negócio (layout, esqueleto) e a semântica dos dados. Também se pode observar na figura 4 que o projeto é supervisionado pela equipe de desenvolvimento, ou seja, todas as tarefas de projeto são supervisionadas pelos desenvolvedores quando se trata de questões relacionadas com o usuário. Ao mesmo tempo, a equipe de desenvolvedores está realizando os diferentes componentes (papel, formulário de dados, projeto) para serem avaliados e construídos com base nas especificações do usuário e de forma interativa (prototipagem). Esses componentes têm o Domínio dos dados de negócio, formado por entidades empresariais que representam dados que suportam as tarefas de negócios. Além disso, depois de fazer a captura de modelos mentais, um design visual realista é feito. No TD-MBUID a apresentação da interface do usuário é dada pela forma, que é capturada em formulário de papel. Assim, (formulário em papel) a estrutura da apresentação da interface do usuário que está ligada à semântica dos dados é descrita.

\subsection{Caracterização da Pesquisa}

Este trabalho se origina por uma pesquisa exploratória que, segundo Barros e Lehfeld [23], busca a aproximação e familiaridade com o tema. Também é descritiva na medida em que descreve as características conhecidas e buscou criar uma teoria explicativa para os fenômenos estudados [24]. Quanto aos procedimentos de coleta de dados, é caracterizada como: Pesquisa bibliográfica para o desenvolvimento do tema usabilidade, das técnicas e metodologia para desenvolvimento de interfaces e da metodologia TD-MBUID.

Para a modelagem do sistema MESO, utilizou-se a metodologia TD-MBUID que agrega técnicas de usabilidade e os diagramas que esta metodologia sugere. Esta etapa foi realizada na Colômbia juntamente com o 
Grupo de Investigação de Sistemas de Informação e Controle Industriais - SINFOCI da Universidad del Quindío. Este grupo tem larga experiência na utilização desta metodologia. Foram utilizados os softwares Rational Rose [25], para a construção do diagrama de dados o qual será demonstrado na próxima seção, e Tobii Studio [26] para a realização de testes com 12 usuários. Os resultados encontrados estão descritos a seguir.

\section{Resultados}

Neste sistema, o foco da usabilidade aparece na construção do questionário e em como gerar intenção no usuário a respondê-lo, ou seja, utilizar o sistema, tendo como prioridade a simplicidade e facilidade na tarefa proposta, gerando intenção de uso, sem a necessidade de profissionais treinados para sua aplicação, facilitando assim seu uso por empresas de todos os portes, mas principalmente nas micro e pequenas empresas. Por este motivo, utilizou-se a metodologia TD-MBUID que une técnicas de usabilidade com o paradigma da orientação a objetos da Linguagem de Modelagem Unificada (UML). Nesta metodologia, elaborou-se um plano de usabilidade conforme segue o escopo:

- Ambiente: escritório.

- Plataforma: Papel e Web.

- Usuário: Gestores de organizações.

- Tarefas: Em um primeiro momento, tarefas sem delegação e com delegação.

Para a modelagem de negócio, seguiu-se a lógica da figura 5, onde se realiza o modelo de negócio e definem-se as tarefas. Em seguida ocorre a análise dos modelos mentais do usuário pelos especialistas e criam-se protótipos em papel para capturar o diálogo externo.

Figura 5: Lógica para modelagem do negócio

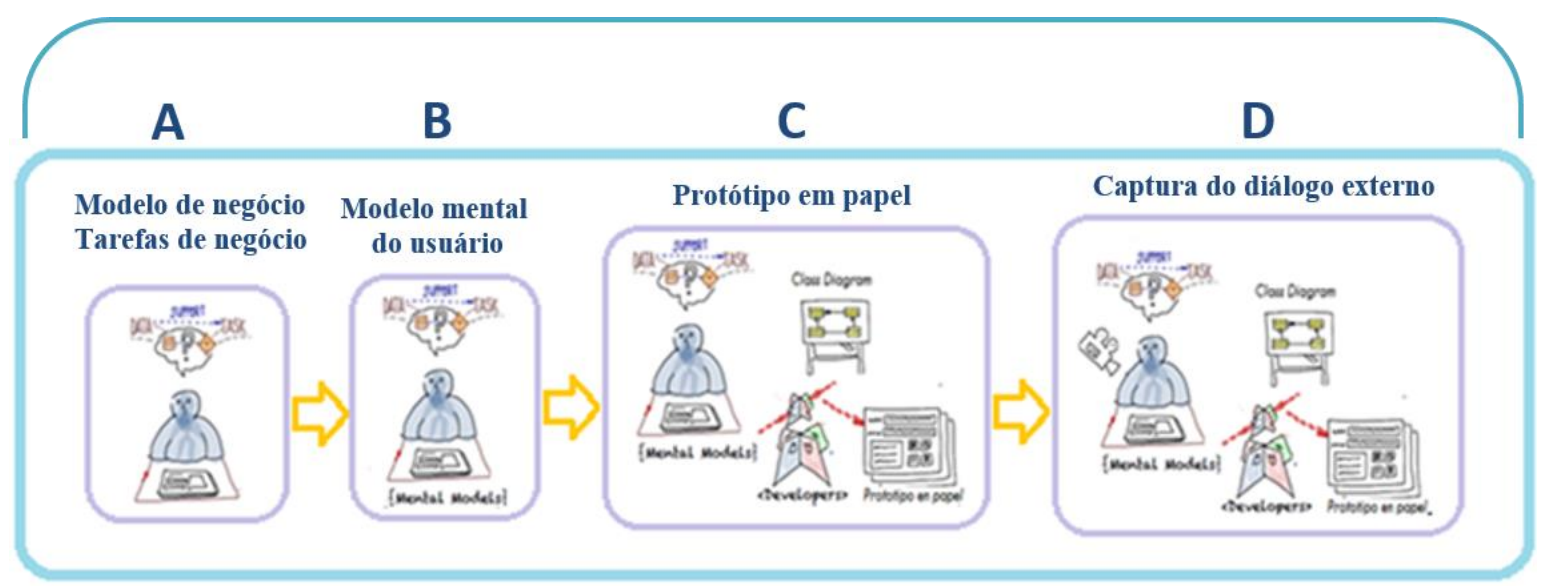

Fonte: Adaptado de Orozco [7].

Na etapa A da figura 5, apresenta-se a relação dos elementos conceituais representativos neste projeto, usando o diagrama de classes do paradigma orientado a objetos. Para a construção do diagrama de dados foi utilizado o programa Rational Rose que é um software da IBM que permite produzir modelos de Linguagem de Modelagem Unificada (UML), além de ser uma ferramenta que permite a criação mais rápida de softwares de qualidade e possibilita a exportação para construção de base dados e testes do modelo. O diagrama foi criado no laboratório SINFOCI que possui a licença do software. Na figura 6, o mapa conceitual construído no início do processo.

Figura 6: Mapa conceitual do modelo MESO primeira versão 


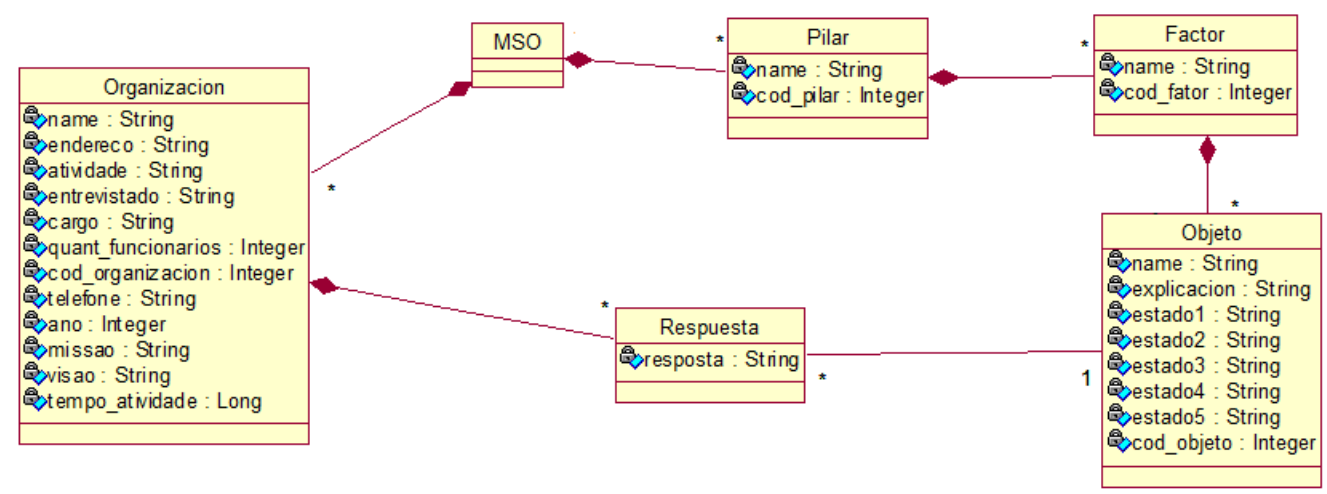

Logo após realizou-se a etapa B da figura 5 que é a captura dos modelos mentais do usuário com integrantes do grupo SINFOCI que não possuíam conhecimento sobre o Modelo MESO ou o sistema proposto. Conforme figura 7, neste momento identificaram-se os conceitos para a construção do sistema e os relacionamentos dos dados necessários e elaborados os protótipos em papel.

Figura 7: Integrantes do SINFOCI realizando os modelos mentais

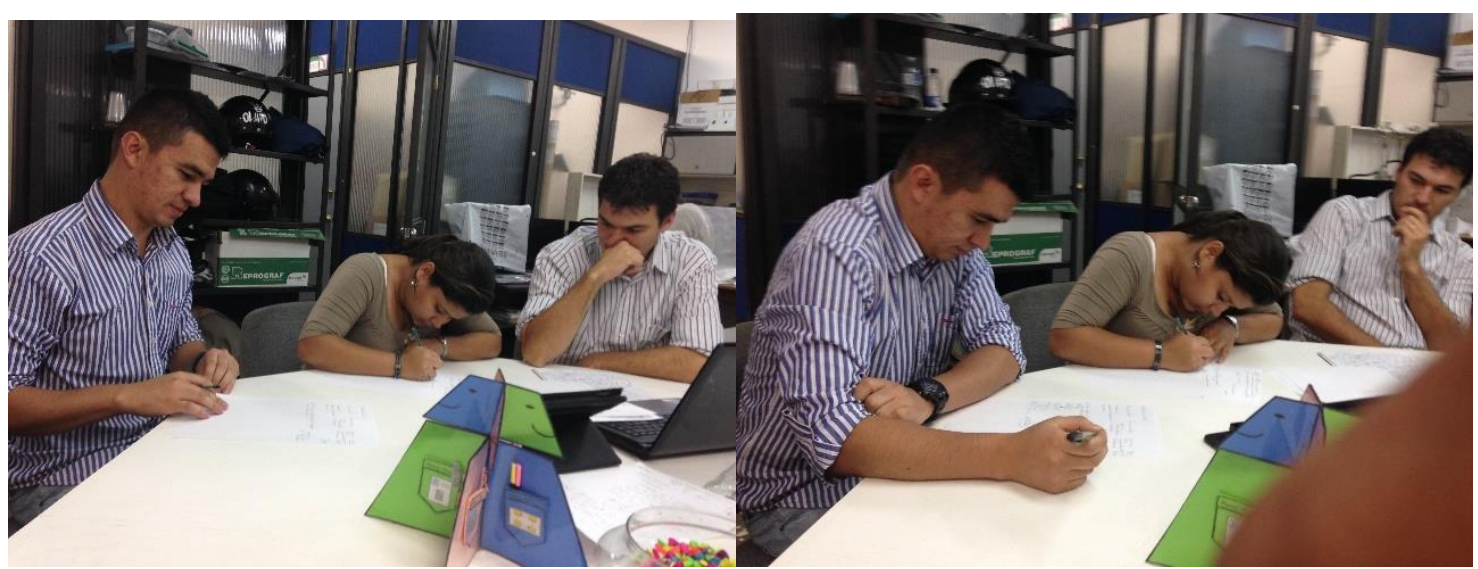

Avaliaram-se as informações do modelo mental do usuário e do domínio (mapa conceitual) para identificar informações do contexto. Esta atividade permitiu enriquecer o modelo de domínio o qual foi novamente modificado para atender às exigências dos usuários, posteriormente foi testado utilizando o software Eclipse Model Framework (EMF), conforme Figura 8. Este framework gera o código a partir do mapa conceitual criado e possibilita testes para verificar a consistência do modelo criado, ou seja, verifica se os dados são suficientes para suportar as tarefas que o usuário vai realizar.

Figura 8: Mapa conceitual testado pelo EMF 


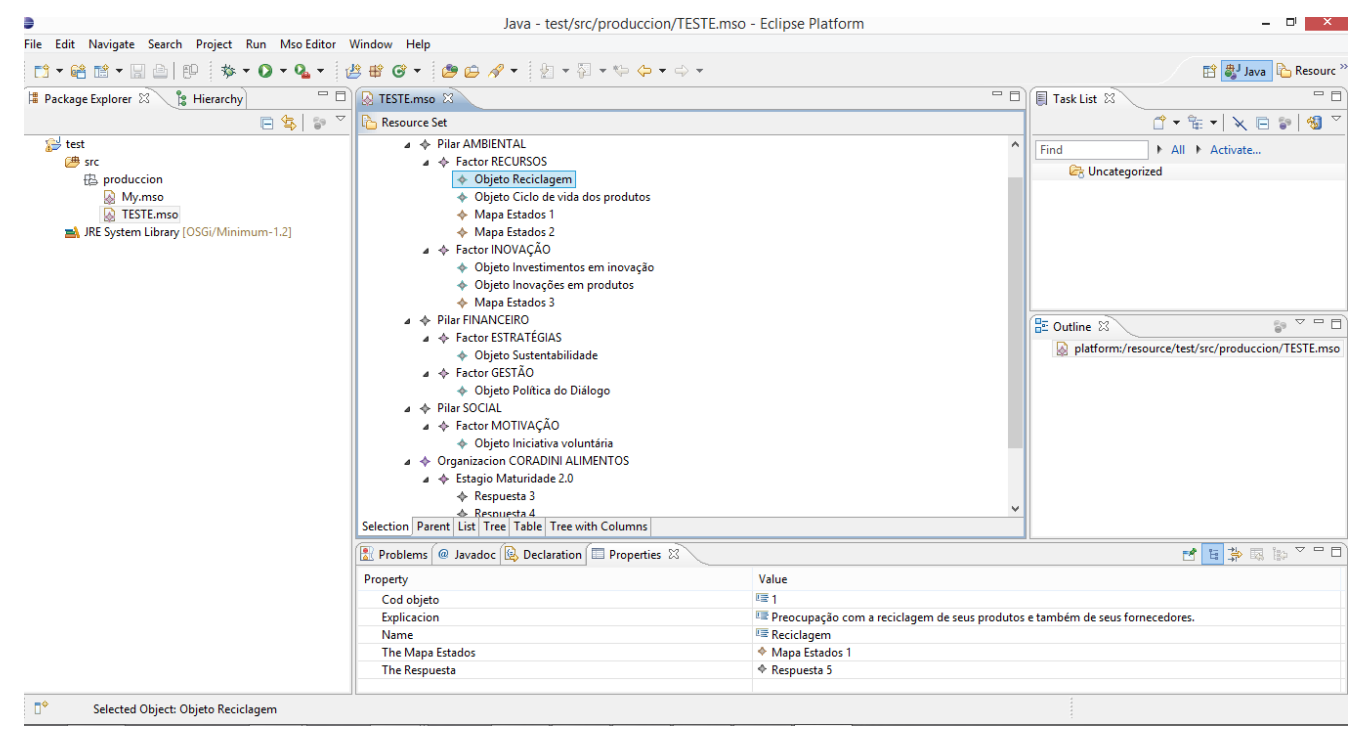

Fonte: Eclipse Model Framework (EMF), 2014.

Segue na figura 9 a nova versão do mapa conceitual na forma de um diagrama de dados, idealizado através dos resultados dos modelos mentais dos usuários.

Figura 9: Mapa conceitual definitivo do modelo MESO.

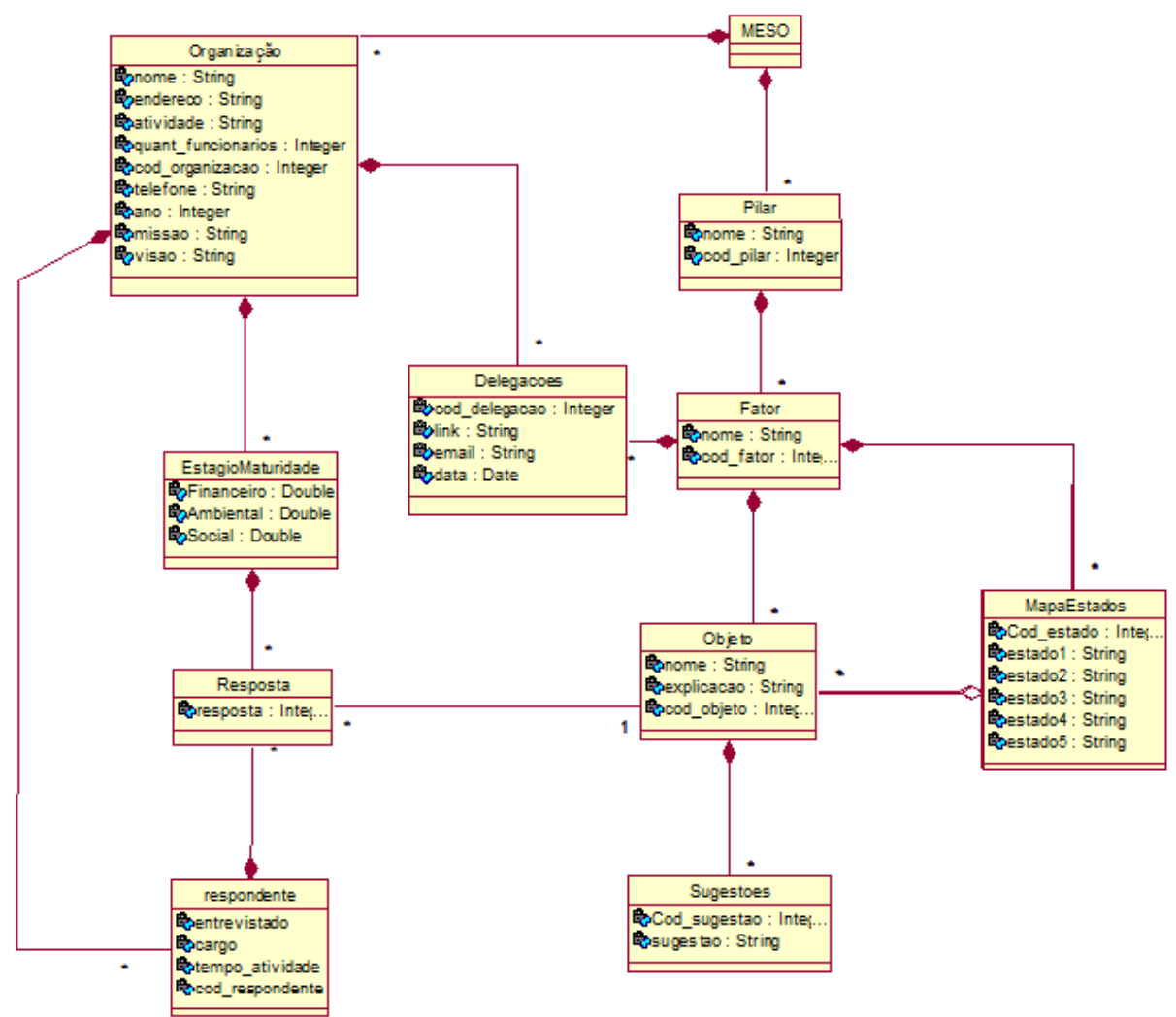

Neste modelo pode-se observar todas as possibilidades do ambiente do sistema computacional MESO, definindo as classes que fazem parte desse modelo com seus respectivos atributos. Na figura 9 do novo modelo conceitual, percebem-se novas classes que facilitam o desenvolvimento do sistema, como as classes "Delegações" e "Respondente" que permitem que mais de um stakeholder possa participar da avaliação da 
organização, e a classe "Sugestões" que permite melhorar o sistema e atualizar as sugestões quando necessário. A classe "Estágio Maturidade" permite análises mais rapidamente e a comparação entre as organizações e, a classe denominada "Mapa Estados" provê um melhoramento na estrutura do modelo permitindo um desenvolvimento mais correto e rápido.

Portanto, os formulários não devem sair dos dados do sistema e sim dos modelos mentais do usuário e da subjetividade do usuário. Um modelo mental é elaborado em três fases ou etapas: a. identificação dos principais conceitos, b. identificação das relações semânticas de dados e forma, c. desenvolvimento da forma.

Um modelo mental está intimamente relacionado com o modelo de formulário de dados (dataform). Um formulário de dados segundo Orozco [7] é um novo modelo especialmente criado para conter ao mesmo tempo e com as mesmas especificações, a semântica dos dados e a semântica da forma que está suportando uma comunicação. A importância do formulário de dados na captação dos modelos mentais é que este elimina a ambiguidade que pode ser gerada pela subjetividade do desenvolvedor ao dar maior importância à subjetividade do usuário.

No modelo de negócios proposto em um ambiente de escritório existem dois atores: um ator principal que é o Gestor da organização que utilizará o sistema e gerará os resultados esperados para avaliar a maturidade da sustentabilidade da organização. O segundo ator que é o facilitador do sistema, que pode ser um ator tecnológico que oriente como realizar as tarefas e gere intenção de utilização do sistema ou um usuário especialista. Para o melhor desenvolvimento do sistema é necessário deixar claro quais são as tarefas ou responsabilidades de cada usuário. Estas são as seguintes: o usuário facilitador deve explicar o sistema, conhecer a metodologia MESO, gerar as sugestões para melhoria, gerar relatório para a gestão, tem a meta de gerar intenção para o usuário utilizar o sistema, e a meta de que o usuário finalize o questionário. Já as responsabilidades do usuário do sistema MESO (usuário Gestor) devem ser: responder ao questionário, interagir com o formulário, repassar perguntas aos outros gestores (quando não souber ou não se sentir em condições para responder uma pergunta).

$\mathrm{Na}$ etapa $\mathrm{C}$ da figura 5 que são os protótipos em papel, estes são a primeira fase do processo de desenho dos formulários, onde são representados os dados do modelo de domínio. Estes protótipos não contêm tipo de botão, menu ou qualquer função que faça referência à tecnologia. Assim, a partir dos modelos mentais dos usuários foram gerados os formulários iniciais gerados pelos usuários através dos modelos mentais.

$\mathrm{Na}$ etapa D da figura 5 a captura completa do diálogo externo gerado a partir de rodadas, novos formulários em papel e discussões até o consenso. Neste último formulário gerado na figura 10 fica clara a identificação do contexto e das tarefas dos usuários, melhorando a semântica dos dados em relação ao questionário, chegando-se no modelo ideal.

Figura 10: Formulário final em papel com a semântica dos dados

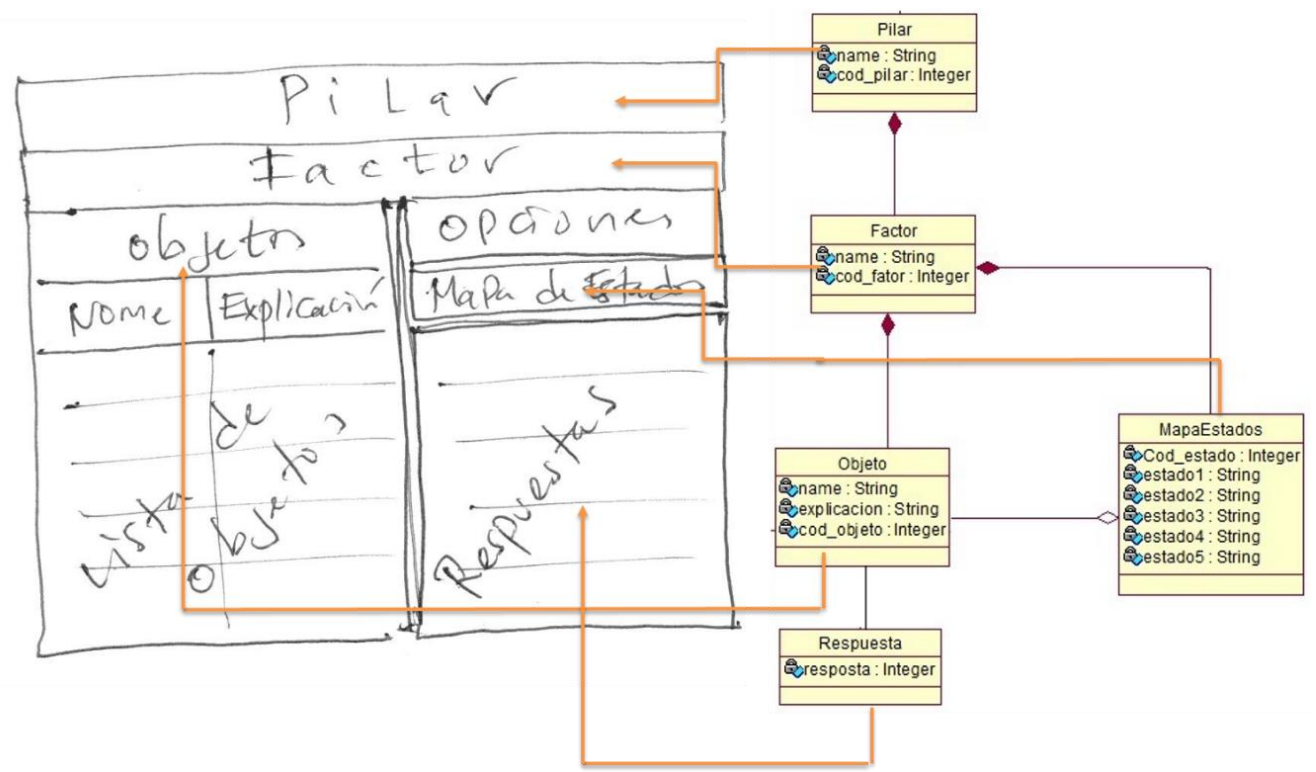

Revista Brasileira de Computação Aplicada (ISSN 2176-6649), Passo Fundo, v. 9, n. 3, p. 2-16, out. 201711 
A figura 10 demonstra a conexão do formulário em papel com o mapa conceitual do sistema, facilitando o seu desenvolvimento e como deve ser a interação do usuário.

Como o foco da usabilidade deste sistema é melhorar a interação do usuário, optou-se por realizar mais uma prova com quatro modelos de questionário gerados com os modelos mentais dos usuários e aplicação do software Tobii Studio que realiza o rastreamento do olhar do usuário.

Esta prova consiste em escolher usuários ao acaso para realizar um teste de interação com o questionário e para isto foi utilizado o software Tobii Studio que permite observar os tempos de reação através da movimentação ocular. Neste software montou-se o experimento, escolhendo os estímulos que serão exibidos, e é através dele que os dados registrados pelas câmeras do equipamento Eye Tracker x2-30 são gravados e visualizados posteriormente, para análise estatística, Neste sistema, pode-se utilizar diversos tipos de estímulos durante o rastreamento do olhar como: textos, imagens, vídeos e páginas WEB. Segue figura 11 com a prova criada no software Tobii Studio.

Figura 11: Teste de questionário no Tobii Studio

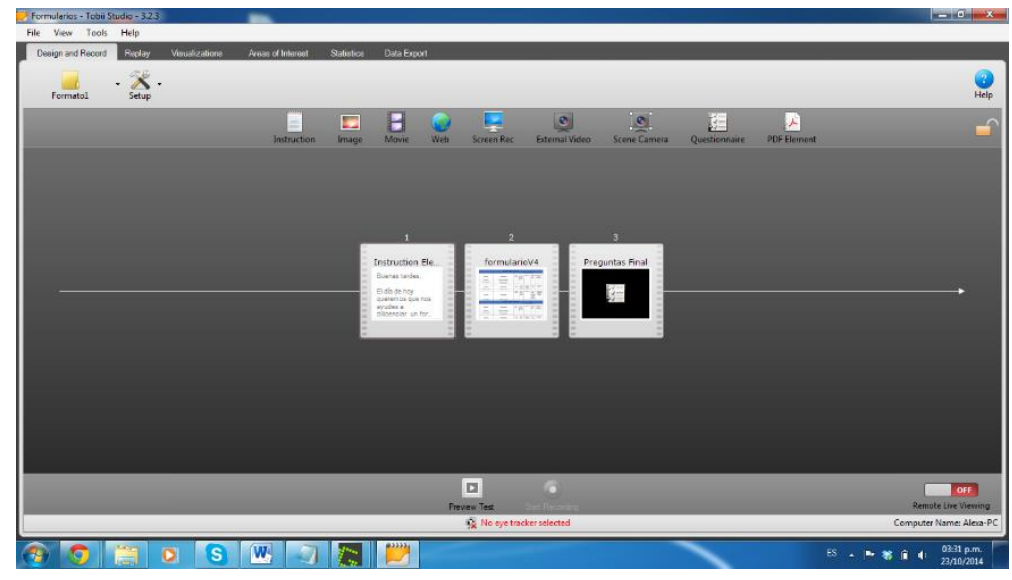

Como se pode visualizar na figura 11 , neste experimento utilizaram-se as imagens dos modelos de questionário, onde:

1- Tela de explicação da tarefa a ser realizada pelo usuário.

2- Formulários em papel (criados a partir dos modelos mentais).

3- Questionário de Usabilidade (prova).

Após o experimento, o software permite visualizar o comportamento ocular dos participantes de diversas formas. Dentre elas:

- Fixações e percurso - Permite rever as fixações do olhar, duração das fixações e as sacadas (movimentos muito rápidos) do movimento ocular do participante.

- Gaze Map (Mapa do traçado do olhar) - Representação animada indicando onde o participante fixou sua atenção. Mostra a ordem das fixações, a duração de cada uma e o trajeto de movimento.

- Heat Maps (Mapas de Calor) - Mostra as áreas onde os participantes fixaram sua atenção com maior frequência (figura 13).

- Bee Swarm (Enxame de abelhas) - Mostra as fixações do olhar de um ou vários participantes durante um período de tempo e ao mesmo tempo.

- Area of Interest (Área de interesse) - Permite verificar estatisticamente a fixação em determinados elementos pré-delimitados (figura 14).

Para realização da prova foram convidados 12 usuários sem conhecimento sobre os formulários em papel e as perguntas foram muito simples, pois a intenção não é avaliar o desempenho e sim o desenho dos formulários. Na figura 12 observa-se um usuário realizando a prova.

Figura 12: usuário nos testes do formulário em papel 


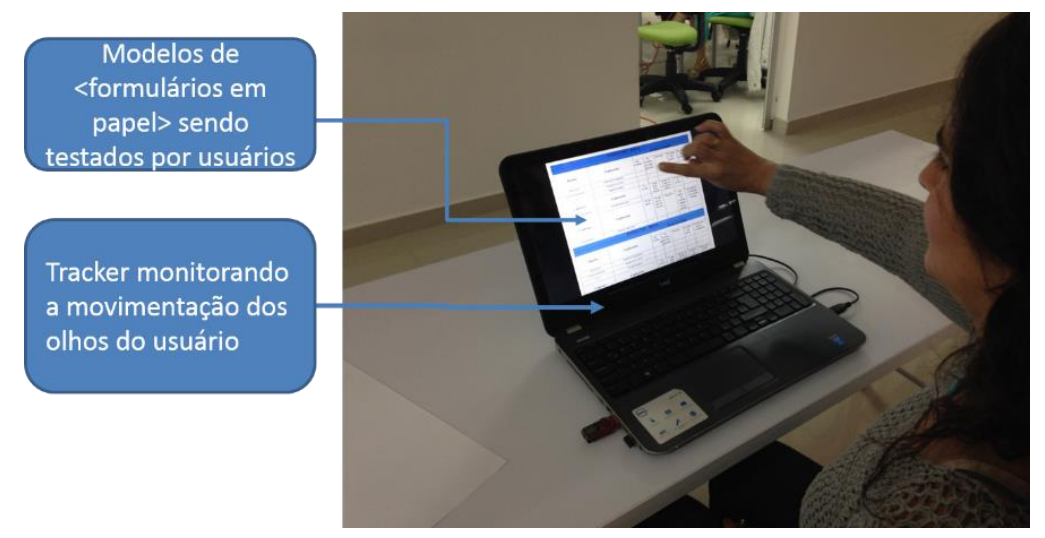

Após a realização das provas com os usuários, buscou-se para análise aplicar duas técnicas em conjunto: área de interesse para marcar aonde se deseja medir estatiscamente à quantidade de vezes que o usuário olhou e o mapa de calor para verificar as áreas que o usuário dedicou sua atenção. Neste experimento, os pontos vermelhos indicam os locais em que o usuário mais fixou seu olhar, ou seja, onde ele concentrou-se para a realização da tarefa. Como se pode observar na figura 13, a imagem 2 foi a que tem um melhor resultado porque os usuários dedicaram seu tempo aos objetos do questionário e não às opções e ao desenho do mesmo, diferente dos outros formulários onde houve um excessivo tempo despendido para entender o formulário e seu objetivo.

Figura 13: Mapas de calor gerados pelo Tobii Studio

1
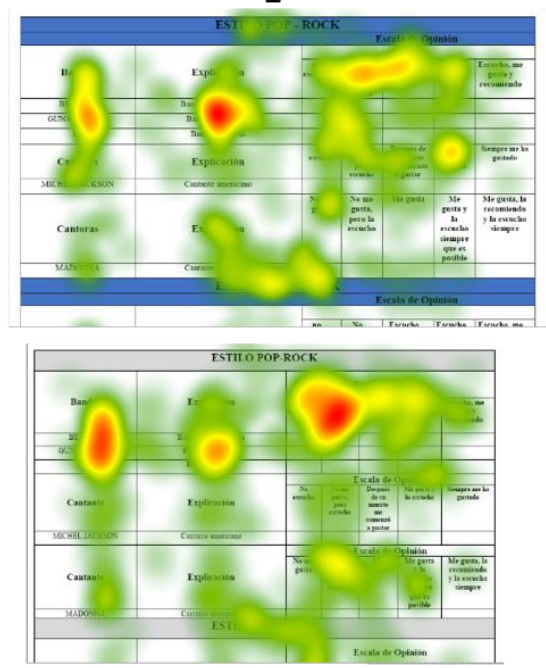

3
2
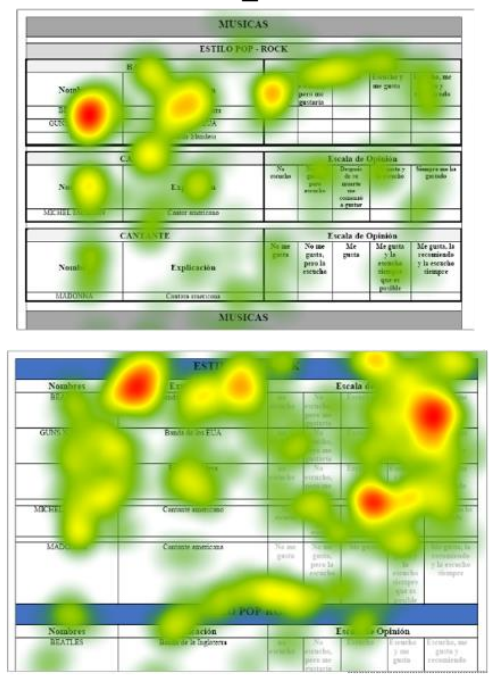

4

Já na figura 14 apresentam-se as áreas de interesse demarcadas, onde se desejou medir estatisticamente, ou seja, quanto tempo o usuário ficou olhando a área demarcada. Obteve-se um tempo menor também no formulário de papel número 2, comprovando o resultado dos mapas de calor. 
Figura 14: Áreas de interesse demarcadas
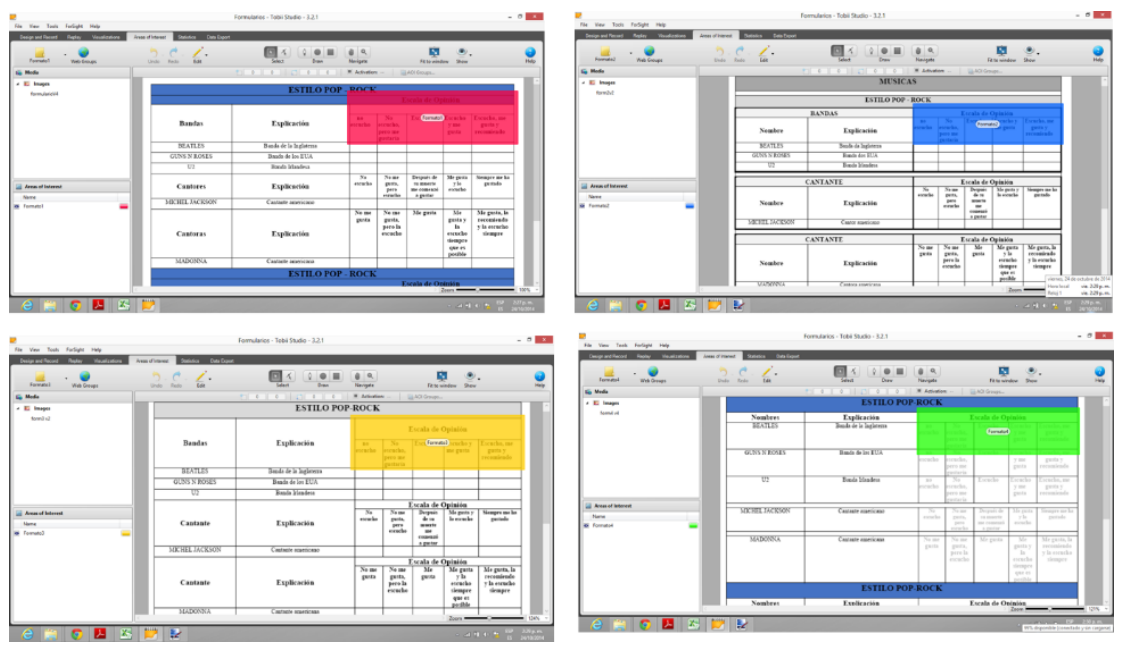

Além dos testes no Tobii também foi importante observar os usuários utilizando os formulários em papel, isso permitiu encontrar erros de desenho em alguns modelos que foram determinantes para que os usuários fixassem seu interesse em informações de desenho e não o que realmente interessava, corroborando com Nielsen [2], ou seja, ficou confuso para os mesmos utilizar e responder o questionário. Com estas provas, pode-se concluir neste experimento que o modelo final em papel (figura 12) desenvolvido através dos modelos mentais foi o que teve melhor resultado, comprovando que técnicas de usabilidade e a metodologia TD-MBUID podem melhorar a interação do usuário com a ferramenta computacional desejada através de um melhor desenho baseado nos modelos mentais e nos modelos de domínio.

\section{Conclusão}

O estudo identificou se técnicas de usabilidade podem auxiliar no desenvolvimento do sistema computacional MESO. O que ficou evidente é que não apenas elas auxiliaram como também permitiram o refinamento de conceitos para sua elaboração. A metodologia TD-MBUID permitiu utilizar o usuário como centro de todo o processo de desenvolvimento do sistema e demonstrou a importância dos modelos mentais do usuário neste processo de desenvolvimento do modelo conceitual e das interfaces, melhorando a usabilidade e a interação, além de utilizar uma técnica inovadora para esse procedimento.

Os resultados deste estudo evidenciam a importância de aliar técnicas de usabilidade no desenvolvimento de softwares e que os testes realizados com o aplicativo Tobii Studio comprovaram que as utilizações dos modelos mentais dos usuários para a elaboração de interfaces auxiliam na usabilidade e na interação dos usuários com as mesmas.

Como sugestão para trabalhos futuros, cita-se utilizar a metodologia TD-MBUID em projetos interdisciplinares do grupo de pesquisa "Rede de pesquisa e Desenvolvimento em Tecnologia Assistiva" vinculada a Universidade da Região da Campanha, URCAMP para avaliar sua contribuição em outros projetos de pesquisa e desenvolvimento.

\section{Agradecimentos}

À UNISC pelo apoio à pesquisa via edital FAP (Fundo de Apoio à Pesquisa) e pela concessão da bolsa de pós-graduação CAPES. 


\section{Referências}

[1] CARNEIRO, Rommel Vieira ; ISHITANI, Lucila Ishitani. Aspectos de usabilidade de mobile learning voltado para usuários com restrições decorrentes da idade. Revista Brasileira de Computação Aplicada. v.6, n.1, p. 81-94, abr. 2014.

[2] NIELSEN, Jakob. Usability 101: Introduction to Usability. Disponível em http://www.nngroup.com/articles/usability-101-introduction-to-usability/ Acesso em: 4 set. 2014.

[3] BEVAN, N. Practical issues in usability measurement. Interactions, v. 13, n. 6, p. 42-43, 2006.

[4] LIMA, Izabel F. et al. Metodologia para avaliação do nível de usabilidade de bibliotecas digitais: Um estudo de caso na biblioteca virtual de saúde. Transinformação, Campinas, V. 25, n.2, p. 135-143, mai/ago., 2013.

[5] FERREIRA, Sueli M.S.P.; REIS, Guilherme. A prática de Arquitetura de Informação de Websites no Brasil. Transinformação, Campinas, v.20, n.3, p. 285-307, set/dez, 2008.

[6] PARK, J. et al. Modeling user experience: A case study on a mobile device. International Journal of Industrial Ergonomics, v. 43, n. 2, p. 187-196, 2013.

[7] OROZCO, W. J. Giraldo, "Marco de desarrollo de sistemas groupware interactivos basado en la integración de procesos y notaciones," Tese de Doutorado en Informática, Universidad de Castilla - La Mancha, España, 2010.

[8] NORMAN, D. A. Interaction design is still an art form. Ergonomics is real engineering. Interactions, v. 13, n. 1, 2006.

[9] NIELSEN, Jakob; LORANGER; Hoa. Usabilidade na web: Projetando websites com qualidade. Rio de Janeiro: Elsevier, 2007.

[10] SILVIA, Patrícia M. O comportamento dos usuários de bibliotecas em sistemas de informação. Transinformação, Campinas, v.20, n.3, p. 255-263, set./dez., 2008.

[11] PREECE, J et. al. Design de interação: além da interação homem-computador. Porto Alegre: Bookman, 2005.

[12] BEVAN, N. et al. Tenuta: Simplified Guidance for Usability and Accessibility. In Proceedings of HCI International. Las Vegas, 2005.

[13] BEVAN, N. Measuring usability as quality of use. Journal of Software Quality, 4, 115-130, 1995.

[14] MARTINS, Ana Isabel et al. Avaliação de Usabilidade: Uma revisão sistemática da literatura. RISTI, Portugal, n. 11, p. 31-43, junho, 2013.

[15] QUARESMA, Manuela; MORAES, Ana Maria. A usabilidade de tarefas típicas de seleção do destino em sistemas de navegação GPS automotivos. Produção, Rio de Janeiro, v. 21, n. 2, p. 329-324, abr./jun. 2011

[16] PARK, J. et al. A Usability Checklist for the Usability Evaluation of Mobile Phone User Interface. International Journal of Human-Computer Interaction, v. 20, n. 3, p. 207-231, 2006.

[17] NIELSEN, Jakob; MACK, Robert. Usability inspection methods. EUA, John Wiley \& Sons, 1994.

[18] PRESSMAN, Roger s. Engenharia de Software: uma abordagem profissional. Porto Alegre: AMGH Editora Ltda, 2011.

[19] SOMMERVILLE, Ian. Engenharia de Software. São Paulo: Pearson Education, 2013.

[20] NASCIMENTO, Ingrid; GASPAR, Wagner; CONTE, Tayana; GADELHA, Bruno; OLIVEIRA, Elaine H. T. Melhor prevenir do que remediar: Avaliando usabilidade e UX de software antes de levá-lo para a sala de aula. V Congresso Brasileiro de Informática na Educação (CBIE 2016), p. 806-815, 2016.

[21] BARBOSA, Fábio; CAMPOS, Dino L.; FIGUEIRÔA, Walter F. M. Correia. Design e Agile: Análise da Metodologia XPlus. InfoDesign. Revista Brasileira de Design da Informação. v. 9, n. 3, p. 153 - 159 , 2012. 
[22] MOLINA, Ana I. et al. CIAT-GUI: A MDE-compliant environment for developing Graphical User Interfaces of information systems. Advances in Engineering Software, n. 52, p. 10-29, 2012.

[23] BARROS, Aidil Jesus da Silveira; LEHFELD, Neide Aparecida de Souza, \& Fundamentos de metodologia científica: um guia para iniciação científica. São Paulo: Pearson Education do Brasil, 2000.

[24] SANTOS, Antonio Raimundo. Metodologia científica: a construção do conhecimento. 3. Ed. Rio de Janeiro: DP\&A, 2000.

[25] RATIONAL ROZE. Disponível em <http://www-03.ibm.com/software/products/pt/rosemod> Acesso em: 5 out. 2014.

[26] TOBII STUDIO. Disponível em <http://www.tobii.com/> Acesso em: 7 out. 2014. 